\title{
Mesenchymal stem cells for therapy in lung diseases
}

\author{
Diana Álvarez ${ }^{1}$ \\ Melanie Levine ${ }^{1,2}$ \\ Mauricio Rojas ${ }^{1,3}$
}

${ }^{1}$ Dorothy P. \& Richard P. Simmons Center for Interstitial Lung Disease, Division of Pulmonary, Allergy and Critical Care Medicine, University of Pittsburgh Medical Center, Pittsburgh, PA, USA

2 Zanvyl Krieger School of Arts \& Sciences, The Johns Hopkins University, Baltimore, MD, USA

${ }^{3}$ McGowan Institute for Regenerative Medicine, Pittsburgh, PA, USA

\section{Address for correspondence:}

Mauricio Rojas

Simmons Center for Interstitial Lung Disease

Division of Pulmonary, Allergy and Critical Care

Medicine

Pittsburgh, PA 15213, USA

Phone: 412-648-8694 - Fax: 412-624-1670

E-mail: rojasm@upmc.edu

\section{Summary}

Lung diseases are among the most devastating illnesses because of their prevalence and simultaneous lack of viable treatment. To advance beyond the current options of mechanical ventilation and transplantation, research in recent years has focused on cell therapy, and of the possibilities, mesenchymal stem cells (MSCs) are the most promising. MSCs have immunomodulatory and secretory effects that work to relieve inflammation and prevent fibrosis in response to the injured lung. Numerous small and large animal models have shown the ameliorating effects of MSCs in diseases such as idiopathic pulmonary fibrosis, chronic obstructive pulmonary disorder, acute respiratory distress syndrome, obstructive sleep apnea, bronchiolitis obliterans, and asthma. Clinical trials for MSC use in some of these are in their early stages, but the encouraging promise of this cell therapy will ensure more trials to come. This review will first discuss MSCs as a population with varied properties and mechanisms of action and then will examine the efforts so far to use MSCs as treatment in various lung diseases.

KEY WORDS: mesenchymal stem cells, lung disease, cell therapy, aging, clinical trials.
Introduction

Lung diseases are some of the most prominent pathologies around the world because of their considerable impact on the population. As the Centers for Disease Control and Prevention reports, chronic lower respiratory diseases constitute the third leading cause of death in the U.S., with, more than 140,000 people dying of such diseases in 2011 (1). Additionally, acute respiratory distress syndrome (ARDS) is the leading cause of death in the intensive care population, with mortality rates between 40 and $60 \%$ (2). Furthermore, progressive and irreversible lung diseases such as idiopathic pulmonary fibrosis (IPF) and chronic obstructive pulmonary disorder (COPD) lack any definitive treatment other than lung transplantation (3). However, the need for transplants far surpasses low donor availability, and each transplant carries risks of rejection for the patient; only about $50 \%$ of lung transplant recipients survive to five years (4).

Non-cancerous lung diseases inhibit normal breathing through the development of fibrosis in the lung tissue or inflammation of airways or general parenchyma, and they include diseases such as COPD, IPF, and ARDS as well as asthma, obstructive sleep apnea, and bronchiolitis obliterans. Obstruction or restriction of the airways causes an increase in airflow resistance or decrease in compliance. Chronic injuries interfere with the healing process that enhances tissue remodeling and fibrosis, increasing the scarring and extracellular matrix components, contributing in that way to the loss of lung function $(5,6)$.

The need for new options is critical, and cell therapy is one of the most promising tools for treatment of lung diseases. Although many properties and mechanisms of action of various types of stem cells are known, details regarding their migration throughout the injured body and their survival mechanisms at the injured tissue are not yet completely understood. Nevertheless, treatment with stem cells constitutes a very good option for patients who would otherwise have to rely on transplantation. The promise of stem cells - specifical-
MSCs participate in lung tissue repair through interaction with stromal and inflammatory cells at the injured tissue. ly, mesenchymal stem cells - in lung diseases will be the subject of this review. The ability of these cells to participate in lung tissue repair through interaction with stromal and inflammatory cells at the injured tissue makes them an undeniable component to the future of lung disease research. 


\section{Identification of Mesenchymal stem cells}

Mesenchymal stem cells (MSCs) are key for tissue repair and regeneration due to their unlimited self-renewal, capacity for clonality, and ability to differentiate in vitro into various specialized cell types that include car-

The recent discovery
of self-renewing, clo-
nogenic, multipotent
lung stem cells with
the ability to form dis-
tal airways and pul-
monary vessels has
exciting implications
for clinical applica-
tion.
tilage, tendon, bone, and adipose tissue $(5,7)$. Thus, MSCs have been identified as key in the management of different chronic pathologies, like type 1 diabetes, graft-versus-host disease, and inflammatory bowel disease (6), and their effects on lung diseases are currently being studied in animal models and clinical trials.

Categorized by origin, MSCs are somatic stem cells. However, the precise characterization of MSCs has been difficult due to the lack of specific surface markers. However, the Mesenchymal and Tissue Stem Cell Committee of the International Society for Cellular Therapy has set minimal criteria to define human MSCs: MSCs when cultured in standard conditions have to be plastic-adherent; they must express adhesion and stromal markers such as CD44, CD105, CD73, and CD90 and lack expression of hematopoietic markers CD45, CD34, CD31, CD14, CD11b, CD19 and CD79 $\alpha$; and the cells must have the ability to differentiate in vitro to chondrocytes, adipocytes, and osteoblasts (8). MSCs have fibroblast-like morphology, so a challenge in selection has been the distinction between MSCs and fibroblasts in culture. Although MSCs share the same markers and have a spindle shape as fibroblasts do, the main characteristic for distinguishing them is the ability of MSCs to forming colonies and the fact that MSCs are multipotent with the ability of selfrenewal, whereas fibroblasts are terminally differentiated cells (9). However, a standard is still lacking to completely distinguish both cell types.

Additionally, the presence of lung-resident stem cells that act as progenitor cells during the response to epithelial injury (10) has led some to contemplate the use of lung-resident stem cells as a treatment option in addition to MSCs. Lung-resident stem cells are unspecialized cells that reside in their own niche within the lung tissue and have the ability to differentiate into specialized cells of the organ. However, due to the high number of cell types that the lung has (more than 40), it has been difficult to recognize the progenitor cells. Some investigations have highlighted type II alveolar epithelial cells, basal cells, and Clue cells as important cells with progenitor functions $(11,12)$. Type II alveolar epithelial cells are essential for maintaining the pulmonary epithelium, but this epithelium has a lower capacity for regeneration and turnover compared to other organs such as skin, making the work of lung resident stem cells insufficient in combating an injury (1113).

The recent discovery (14) of self-renewing, clonogenic, multipotent lung stem cells with the ability to form dis- tal airways and pulmonary vessels has exciting implications for clinical application. These cells would certainly be ideal for therapy in the damaged lung, but the novelty of this discovery means that little is known about lung stem cells so more investigation is needed. Additionally, there has been special attention paid to the potential use of induced pluripotent stem cells in lung diseases, but these and progenitor cells will not fall under the scope of this review.

\section{Mechanisms of action of MSCs}

It is thought that due to the close relationship between the lung epithelium and the stromal tissue, MSCs may play a role in the maintenance of the epithelium (6). Although some mechanisms are still being investigated, a number of studies have supported the role of MSCs in immunomodulation and the anti-inflammatory response. Mechanisms that include cell-cell contact and secretion of mediators (15)

may play a key role in tissue regeneration due to the ability of MSCs to induce T-cell apoptosis that triggers macrophages to increase levels of TGF- $\beta$ (16). Additionally, MSCs secrete IL-10, suppressing T-cell responses and inducing regulatory T-cells and thus, promoting mucosal tolerance (17). Growth factors secreted by the MSCs that are generated through inflammatory stimuli activate the immunosuppressive capacity of MSCs. Additionally, MSCs secrete chemokine and adhesion molecules that act in a synergistic manner with substances like nitric oxide or indoleamine 2,3 dioxygenase to suppress the immune system (6), and they can further protect against acute lung injury by secreting microvesicles that contain mitochondria, which are transferred to the alveolar epithelium (18). The application of these effects to different lung diseases is shown in Figure 1.

\section{Other properties of MSCs}

Another crucial property of MSCs is the low expression of HLA I and HLA II, which provide poor immunogenicity (10), causing MSCs to avoid the cytotoxic $T$ cells and natural killer cells. This confers to MSCs a form of immunoprivilege and with it, the ability to be transplanted without generating an immune response in patients with a competent immune system (15). However, these properties are lost with differentiation (19).

The ability of MSCs to contribute to the restoration of injured tissue has been proven not only by their im- 


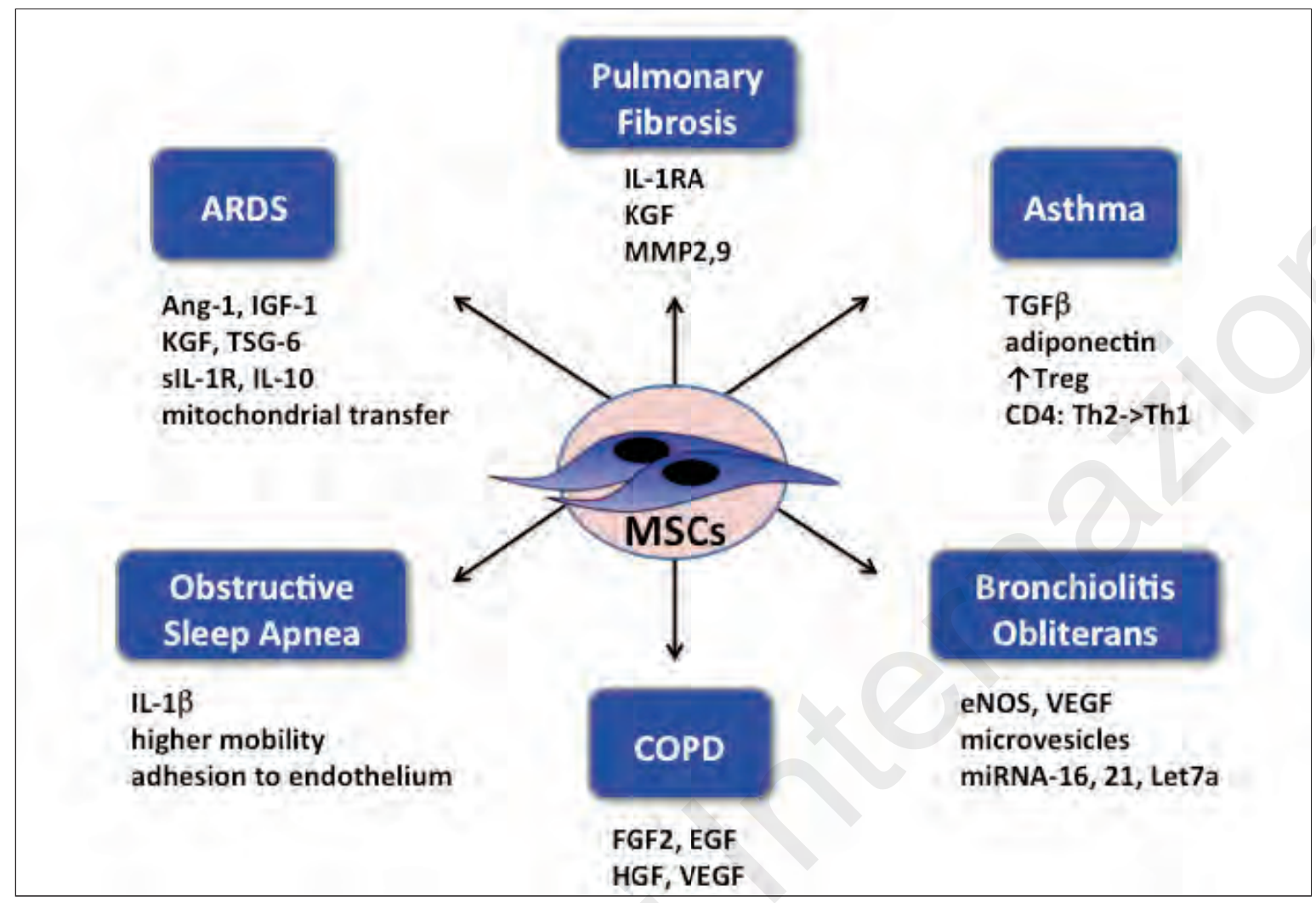

Figure 1 - MSCs have a variety of effects on lung diseases including secretion of growth factors, cytokines, and microvesicles.

munomodulatory and anti-inflammatory properties, but also by their capacities for mitochondrial transfer, as mitochondrial dysfunction constitutes an important facet of apoptosis, cellular senescence, and cell proliferation. Islam et al. proved that ATP concentration in the host pulmonary alveoli increases after the instillation of MSCs, contributing to energetic restoration and protection in the acute phase of lung injury, and supporting the presence of mitochondrial transfer from the MSC to the host cell in a lipopolysaccharide acute lung injury model (18).

The amelioration of lung injury has been demonstrated by our group through several murine models of injury. We evaluated the functions of bone marrow-derived MSCs in myelosupressed and normal mice and showed that in a bleomycin model, only one third of the immunosupressed mice survived after 14 days. However, after administration of MSCs six hours after the lung injury there was a $100 \%$ rate of survival, and the lung injury was minimal in the immunocompetent mice. We also showed that with MSC administration, the levels of G-CSF and GM-CSF increased, causing mobilization of stem cells from pools (20).

We have also demonstrated the ability of MSCs to migrate to the injured lung. By using a pore membrane and fluorescence microscopy, we culturing mice lung cells with green fluorescent protein and MSCs after a single dose of LPS or saline. The results obtained showed migration of MSCs toward the cell suspensions (16).

\section{Aging and MSCs}

Because the incidence of both acute diseases such as ARDS and chronic diseases such as COPD and IPF increases with age, a special interest has emerged in the study of aging and its relation not only to these pathologies but also to the use of MSCs as therapy (21).

Some elements present in normal processes of aging, such as oxidative stress and abnormal telomere shortening, are important deleterious effects of tobacco smoking on pulmonary parenchyma $(3,22)$. It is well known that an appropriate telomere length is crucial for the survival of stem cells, and this has been supported by the expression of telomerase in type II alveolar epithelial cells following alveolar

damage (22).

Other factors in aging include changes in the composition of the extracellular matrix and changes related to the decrease in cardiac output that contribute to lower arterial pO2 and loss of lung volume. All these
The decrease in the expression of several cytokine and chemokine receptors associated with aging are important factors in the migration and activation of MSCs. factors, associated with a decrease in the adrenergic control of cardiovascular function according to normal aging, lead to a decrease in the response to sepsis and might affect the migration of MSCs to the injured tissue (21). 
Publications from our group and others have shown that multiple injuries lead to premature aging through continuous stem cell activation and depletion. We demonstrated as well that the decrease in the expression of several cytokine and chemokine receptors associated with aging are important factors in the migration and activation of MSCs $(23,24)$.

\section{MSCs in acute lung diseases}

ARDS has a prevalence estimated at $22 \%$ and an inhospital mortality close to $40 \%$ (25), making it the leading cause of death for intensive care unit patients (2). Lung conditions and systemic diseases that affect the lung through the bloodstream are associated with the development of ARDS, and the disease encompasses an increase in alveolar and vascular permeability (mediated by endothelin-1, phospholipase A2, and angiotensin-2), loss of epithelial integrity, influx of inflammatory cells (IL-1 $\beta$, TNF- $\alpha$, and IL-6), and formation of hyaline membranes in a complex process that frequently ends in multiple organ failure as a cause of death (16).

The Berlin definition (26) set the new diagnosis criteria for ARDS based on: timing (within one week of clinical insult or new or worsening respiratory symptoms), radiography (bilateral opacities not fully explained by effusions, lobar/lung collapse, or nodules), origin of edema (respiratory failure not fully explained by cardiac failure or fluid overload), and oxygenation impairment (mild: $200 \mathrm{mmHg}<\mathrm{PaO} 2 / \mathrm{FiO} 2<300 \mathrm{mmHg}$, moderate: $100 \mathrm{mmHg}<\mathrm{PaO} 2 / \mathrm{FiO} 2200<\mathrm{mmHg}$, and severe: $\mathrm{PaO} 2 / \mathrm{FiO} 2<100 \mathrm{mmHg}$ ). Thus, according to this definition, the term "acute lung injury" as used previously now falls in the category of moderate ARDS $(16,26)$.

Despite the increased interest in reaching a curative treatment for this condition, the current therapies focus on supportive care techniques of lung protective ventilation and conservative fluid strategy (27), and there is not a proven therapy to reduce the severity of lung injury or to improve the outcomes. Thus, the literature has focused on different options that contribute to tissue repair - not only to support of injured lungs opening in this way an opportunity for the therapeutic use of MSCs.

Different studies have demonstrated the proliferative potential of MSCs through the secretion of cytokines and growth factors using murine (28) and large animal $(29,30)$ models of endotoxin-induced ARDS. These experiments revealed a decrease in proinflammatory cytokines, increase in anti-inflammatory cytokines, histological decrease in lung injury, and overall increase in survival rates $(16,20,28-34)$. Our group has demonstrated that infusion of MSCs prevents the inflammatory response to endotoxin and attenuates the lung injury, protecting as well against pulmonary edema (7). Furthermore, MSCs regulate the inflammatory signaling process in injured lung cells by decreasing IL-1 $\beta$, TNF- $\alpha$, and IL- 6 among others $(7,33)$. To improve MSC efficacy, the cells can be preactivated in vitro through stimulation with serum from ARDS patients. In a murine model, use of activated MSCs resulted in reduced histological lung inflammation scores and lung vascular permeability as well as decreased levels of inflammatory cells compared with treatment with nonstimulated MSCs (35).

\section{MSCs in chronic lung diseases}

\section{Chronic pulmonary obstructive disease}

Chronic pulmonary obstructive disease (COPD) is the third leading cause of death worldwide (36) with smoking as the principal preventable risk factor. Emphysema, characterized by a loss of alveolar architecture, is a key component present in COPD, and although the pathogenesis of emphysema is still unclear (37), inactivation of vascular endothelial growth factor (VEGF) is crucial in the development of this condition (38). MSCs act in the emphysematous lung through suppression of apoptosis $(39,40)$, downregulation of proinflammatory cytokines TNF- $\alpha$, IL-1, and IL-6, and upregulation of VEGF and TGF $\beta-1$ (40). In a clinical trial recently conducted by Weiss et al., 62
Attenuation of asthmatic injury by MSCs will likely come through immunological mechanisms, rather than engraftment in the lung airway or alveolar epithelium. patients were given doses of either allogeneic MSCs or a placebo, demonstrating the safety of cell therapy for COPD. A decrease in the C-reactive protein in the MSC treatment suggests a role for MSCs in the modulation of the inflammatory pathway. However, there were not significant differences in pulmonary function test or quality of life, so future investigations should be forthcoming $(41,42)$.

\section{Idiopathic pulmonary fibrosis}

Idiopathic pulmonary fibrosis (IPF), the most common type of interstitial lung disease, is irreversible, progressive, and has a poor prognosis. The prevalence is around 27.9 cases per 100,000 habitants, increasing with age, and the prevalence is greater among males than females (43). The complete understanding of the pathogenesis of IPF still remains uncertain; however, an abnormal activation of regenerative processes has been implicated by the increase in the deposition of fibroblasts and myofibroblasts (22).

Different bleomycin models in mice have been conducted, including studies from our group, where early treatment with MSCs has shown reductions in collagen deposition and expression of matrix metalloproteinases 2 and 9, decreases in epithelial damage, and improved survival rates $(20,31,44,45)$.

Confidence in the use of MSCs as therapy for IPF patients has progressed to the clinical trial phase, with one completed phase I trial and several in the recruiting stage (clinicaltrials.gov).

\section{Bronchiolitis obliterans}

Brochiolitis obliterans (BO) is one of the main concerns 
in pulmonary transplantation because of the poor prognosis that it entails, affecting around $50 \%$ of all the lung transplant recipients, with a mean survival time of 3 years (46). It is believed to be a form of chronic rejection in which management with high doses of immunosuppressive agents seems to be the only available therapy, even accounting for the deleterious effects often observed with the use of these agents (47).

$\mathrm{BO}$ is characterized by the presence of a patchy submucosal fibrosis involving the respiratory bronchioles, which leads to obliteration of the distal airways by inflammatory infiltrates, fibroblasts, collagen, and matrix deposition. The damage to the airways and decrease in the total surface area for gas exchange induces chronic pulmonary dysfunction $(47,48)$. Although the complete mechanisms that participate in this pathology are not fully understood, fibrocytes are thought to play a key role, so they may be useful as a marker in future studies (49).

The beneficial effects of MSCs seen with diseases other than lung pathologies suggests that they may be beneficial in treating $\mathrm{BO}$. In a mouse model of heterotopic tracheal transplantation, our group demonstrated the positive effects by bone marrow-derived MSCs on the attenuation of airway obliteration (26), and another group has demonstrated a similar protective effect by placenta-derived MSCs in the heterotopic tracheal transplantation (50). In murine orthotopic tracheal transplantation, MSCs protected as well against allograft rejection, further supporting the promising role of stem cells in $\mathrm{BO}(51)$.

\section{Asthma}

The World Health Organization estimates that 235 million people worldwide currently suffer from asthma (52), and the allergen-driven disease is a likely target of therapy by MSC immunomodulation. Asthma is characterized by inflammation and hyperresponsiveness of the airway, and airway wall remodeling includes epithelial injury, thickening of the reticular basement membrane, and increases in airway smooth muscle mass (53).

Attenuation of asthmatic injury by MSCs will likely come through immunological mechanisms, rather than engraftment in the lung airway or alveolar epithelium, which is a rare occurrence (54). Several studies have shown the efficacy of MSC use in animal models of asthma and support the anti-inflammatory mechanisms of the cells. In a murine ovalbumin model of chronic asthma, IL-5, IL-13, IgE, and iNOS were all decreased, as were eosinophil levels (55). In a murine ragweed model, airway mucus and eosinophil levels were decreased, and IL-4 and IL-13 levels were reduced by treatment with MSCs (56). The study also showed that the MSC-treated animals recruited more anti-inflammatory regulatory $\mathrm{T}$ cells to the lungs.

\section{Obstructive sleep apnea}

Obstructive sleep apnea (OSA) is a breathing disorder in which increased upper airway collapsibility leads to recurrent intrathoracic pressure swings and hypoxia. The recurrent apneas disrupt normal sleep patterns, and the oxidative stress and inflammation induced by hypoxia can have long-term effects of hypertension, ischemia, cerebrovascular disease, and heart failure (57).

The research on MSCs is limited, but Carreras et al. have shown that in a rat model of OSA, the recurrent apneas caused early release of MSCs into the circulating blood (58), that the MSCs had higher mobility and increased adhesion to endothelial cells (59), and that MSCs contributed to the anti-inflammatory response prompted by the inflammation of the disease by decreasing levels of IL-1 $\beta$ (60). In a study that found that OSA induces atrial fibrosis, treatment with MSCs was also found to prevent the increase in IL-1 $\beta$ (61). The greatest benefit of MSC therapy in OSA, then, appears to be the anti-inflammatory effect that can counter the damaging inflammation of the obstructed airways.

\section{Conclusion}

It is clear that MSCs are a promising therapy for lung diseases. The success of preclinical animal studies has proven the ameliorating effects on MSCs on a number of lung diseases, through mechanisms that include decreasing levels of pro-inflammatory cytokine, suppressing apoptosis, and secreting growth factors and mitochondria. Studies to better understand the aspects that still remain unknown are ongoing and will contribute to future treatment options. As clinical trials progress in COPD and IPF, we will soon know more about this potentially life-saving therapy.

\section{References}

1. Hoyert DL, Xu J. Deaths: preliminary data for 2011. National vital statistics reports. 2012;61(6):1-51.

2. Hayes M, Curley G, Ansari B, Laffey JG. Clinical review: Stem cell therapies for acute lung injury/acute respiratory distress syndrome-hope or hype. Crit Care. 2012;16(2):205.

3. Huleihel L, Levine M, Rojas M. The potential of cellbased therapy in lung diseases. Expert opinion on biological therapy. 2013;13(10):1429-40.

4. What Are the Risks of Lung Transplant? [Web]. National Heart, Lung, and Blood Institute; [updated 1 May 20113 July 2014]. Available from: http://www. nhlbi.nih.gov/health/health-topics/topics/lungtxp/ risks.html.

5. Kolios G, Moodley Y. Introduction to stem cells and regenerative medicine. Respiration. 2012;85(1):310.

6. Shi Y, Su J, Roberts Al, Shou P, Rabson AB, Ren G. How mesenchymal stem cells interact with tissue immune responses. Trends Immunol. 2012;33(3): 136-43.

7. Iyer S, Co C, Rojas M. Mesenchymal stem cells and inflammatory lung diseases. Panminerva medica. 2009;51(1):5.

8. Dominici M, Le Blanc K, Mueller I, Slaper-Cortenbach I, Marini F, Krause D, et al. Minimal criteria for defin- 
ing multipotent mesenchymal stromal cells. The International Society for Cellular Therapy position statement. Cytotherapy. 2006;8(4):315-7. doi: 10.1080/14653240600855905. PubMed PMID: 22036764.

9. Alt E, Yan $Y$, Gehmert S, Song $Y H$, Altman A, Gehmert S, et al. Fibroblasts share mesenchymal phenotypes with stem cells, but lack their differentiation and colony-forming potential. Biol Cell. 2011;103(4):197-208.

10. Sinclair K, Yerkovich ST, Chambers DC. Mesenchymal stem cells and the lung. Respirology. 2013;18(3):397-411.

11. Kotton DN, Summer R, Fine A. Lung stem cells: new paradigms. Experimental hematology. 2004;32(4): 340-3.

12. Loebinger MR, Janes SM. Stem cells for lung disease. CHEST Journal. 2007;132(1):279-85.

13. Royce SG, Moodley Y, Samuel CS. Novel therapeutic strategies for lung disorders associated with airway remodelling and fibrosis. Pharmacol Ther. 2014;141(3):250-60.

14. Kajstura J, Rota M, Hall SR, Hosoda T, D'Amario D, Sanada F, et al. Evidence for human lung stem cells. New Engl J Med. 2011;364(19):1795-806.

15. Sueblinvong V, Weiss DJ. Cell therapy approaches for lung diseases: current status. Curr Opin Pharm. 2009;9(3):268-73.

16. Cárdenes N, Cáceres E, Romagnoli M, Rojas M. Mesenchymal stem cells: a promising therapy for the acute respiratory distress syndrome. Respiration. 2013;85(4):267-78.

17. Le Blanc K, Mougiakakos D. Multipotent mesenchymal stromal cells and the innate immune system. Nature Reviews Immunology. 2012;12(5):383-96.

18. Islam MN, Das SR, Emin MT, Wei M, Sun L, Westphalen $\mathrm{K}$, et al. Mitochondrial transfer from bone-marrow-derived stromal cells to pulmonary alveoli protects against acute lung injury. Nat Med. 2012;18(5):759-65.

19. Sabir HJ, Nehlin JO, Qanie D, Harkness L, Prokhorova TA, Blagoev B, et al. Separate Developmental Programs for HLA-A and -B Cell Surface Expression during Differentiation from Embryonic Stem Cells to Lymphocytes, Adipocytes and Osteoblasts. PLoS ONE. 2013;8(1):1-9.

20. Rojas M, Xu J, Woods CR, Mora AL, Spears W, Roman J, et al. Bone marrow-derived mesenchymal stem cells in repair of the injured lung. Am J Respir Cell Mol Biol. 2005;33(2):145-52.

21. Mora AL, Rojas M. Aging and lung injury repair: a role for bone marrow derived mesenchymal stem cells. J Cell Biochem. 2008;105(3):641-7.

22. Chilosi M, Doglioni C, Murer B. Epithelial stem cell exhaustion in the pathogenesis of idiopathic pulmonary fibrosis. Sarcoidosis vasculitis and diffuse lung disease. 2010;27(1):7-18.

23. Bustos ML, Huleihel L, Kapetanaki MG, Lino-Cardenas CL, Mroz L, Ellis BM, et al. Aging Mesenchymal Stem Cells Fail to Protect Because of Impaired Migration and Antiinflammatory Response. Am J Respir Crit Care Med. 2014;189(7):787-98.
24. Kapetanaki MG, Mora AL, Rojas M. Influence of age on wound healing and fibrosis. The Journal of pathology. 2013;229(2):310-22.

25. Rincon F, Ghosh S, Dey S, Maltenfort M, Vibbert M, Urtecho J, et al. Impact of acute lung injury and acute respiratory distress syndrome after traumatic brain injury in the United States. Neurosurgery. 2012;71(4):795-803.

26. Force ADT. Acute respiratory distress syndrome. Jama. 2012;307(23):2526-33.

27. Cribbs SK, Matthay MA, Martin GS. Stem cells in sepsis and acute lung injury. Critical care medicine. 2010;38(12):2379.

28. Xu J, Woods CR, Mora AL, Joodi R, Brigham KL, lyer $\mathrm{S}$, et al. Prevention of endotoxin-induced systemic response by bone marrow-derived mesenchymal stem cells in mice. American Journal of Physiology - Lung Cellular and Molecular Physiology. 2007;293(1): L131-L41. doi: 10.1152/ajplung.00431.2006.

29. Rojas M, Cárdenes N, Kocyildirim E, Tedrow JR, Cáceres E, Deans R, et al. Human adult bone marrow-derived stem cells decrease severity of lipopolysaccharide-induced acute respiratory distress syndrome in sheep. Stem cell research \& therapy. 2014;5(2):42.

30. Rojas M, Parker RE, Thorn N, Corredor C, lyer SS, Bueno $\mathrm{M}$, et al. Infusion of freshly isolated autologous bone marrow derived nuclear cells prevents endotoxin-induced lung injury in an ex-vivo perfused swine model. Stem Cell Research \& Therapy. 2013;4(2):26.

31. Gotts JE, Matthay MA. Mesenchymal stem cells and acute lung injury. Critical care clinics. 2011;27(3):71933.

32. Gupta N, Su X, Popov B, Lee JW, Serikov V, Matthay MA. Intrapulmonary Delivery of Bone Marrow-Derived Mesenchymal Stem Cells Improves Survival and Attenuates Endotoxin-Induced Acute Lung Injury in Mice. The Journal of Immunology. 2007;179(3): 1855-63.

33. Ortiz LA, DuTreil M, Fattman C, Pandey AC, Torres $\mathrm{G}$, Go K, et al. Interleukin 1 receptor antagonist mediates the antiinflammatory and antifibrotic effect of mesenchymal stem cells during lung injury. Proceedings of the National Academy of Sciences. 2007;104(26):11002-7.

34. Zhao F, Zhang Y, Liu Y, Zhou J, Li Z, Wu C, et al., editors. Therapeutic effects of bone marrow-derived mesenchymal stem cells engraftment on bleomycininduced lung injury in rats. Transplantation proceedings; 2008: Elsevier.

35. Bustos ML, Huleihel L, Meyer EM, Donnenberg AD, Donnenberg VS, Sciurba JD, et al. Activation of human mesenchymal stem cells impacts their therapeutic abilities in lung injury by increasing interleukin (IL)10 and IL-1RN levels. Stem cells translational medicine. 2013;2(11):884.

36. The top 10 causes of death: Fact sheet $N^{\circ} 310$ : World Health Organization; 2014 [updated May 20147 July 2014]. Available from: http://www.who.int/mediacentre/factsheets/fs310/en/.

37. Mora AL, Rojas M. Adult stem cells for chronic lung diseases. Respirology. 2013;18(7):1041-6. 
38. Zhen G, Xue Z, Zhao J, Gu N, Tang Z, Xu Y, et al. Mesenchymal stem cell transplantation increases expression of vascular endothelial growth factor in papain-induced emphysematous lungs and inhibits apoptosis of lung cells. Cytotherapy. 2010;12(5):60514.

39. Zhen G, Liu H, Gu N, Zhang H, Xu Y, Zhang Z. Mesenchymal stem cells transplantation protects against rat pulmonary emphysema. Frontiers in bioscience: a journal and virtual library. 2007;13:3415-22.

40. Guan XJ, Song L, Han FF, Cui ZL, Chen X, Guo XJ, et al. Mesenchymal stem cells protect cigarette smoke-damaged lung and pulmonary function partly via VEGF-VEGF receptors. J Cell Biochem. 2013;114(2):323-35.

41. Sueblinvong V, Weiss DJ. Stem cells and cell therapy approaches in lung biology and diseases. Translational Research. 2010;156(3):188-205.

42. Weiss DJ, Casaburi R, Flannery R, LeRoux-Williams M, Tashkin DP. A placebo-controlled, randomized trial of mesenchymal stem cells in copd. CHEST Journal. 2013;143(6):1590-8. doi: 10.1378/chest.122094.

43. Nalysnyk L, Cid-Ruzafa J, Rotella P, Esser D. Incidence and prevalence of idiopathic pulmonary fibrosis: review of the literature. European Respiratory Review. 2012;21(126):355-61.

44. Antunes MA, Laffey JG, Pelosi P, Rocco PR. Mesenchymal Stem Cell Trials for Pulmonary Diseases. J Cell Biochem. 2014;115(6):1023-32.

45. Moodley Y, Atienza D, Manuelpillai U, Samuel CS, Tchongue J, llancheran S, et al. Human umbilical cord mesenchymal stem cells reduce fibrosis of bleomycin-induced lung injury. The American journal of pathology. 2009;175(1):303-13.

46. Martinu T, Palmer SM, Ortiz LA. Lung-resident Mesenchymal Stromal Cells: A New Player in Post-Transplant Bronchiolitis Obliterans Syndrome? Am J Respir Crit Care Med. 2011;183(8):968-70.

47. Grove DA, Xu J, Joodi R, Torres-Gonzales E, Neujahr D, Mora AL, et al. Attenuation of early airway obstruction by mesenchymal stem cells in a murine model of heterotopic tracheal transplantation. The Journal of Heart and Lung Transplantation. $2011 ; 30(3): 341-50$.

48. Todd JL, Palmer SM. Bronchiolitis obliterans syndrome: the final frontier for lung transplantation. CHEST Journal. 2011;140(2):502-8.

49. LaPar DJ, Burdick MD, Emaminia A, Harris DA, Strieter BA, Liu L, et al. Circulating fibrocytes correlate with bronchiolitis obliterans syndrome development after lung transplantation: a novel clinical biomarker. The Annals of thoracic surgery. 2011;92(2):470-7.

50. Zhao Y, Gillen JR, Harris DA, Kron IL, Murphy MP, Lau CL. Treatment with placenta-derived mesenchymal stem cells mitigates development of bronchiolitis obliterans in a murine model. The Journal of Thoracic and Cardiovascular Surgery. 2014;147(5): 1668-77.e5. doi: http://dx.doi.org/10.1016/j.jtcvs. 2013.09.041.
51. Guo Z, Zhou X, Li J, Meng Q, Cao H, Kang L, et al. Mesenchymal stem cells reprogram host macrophages to attenuate obliterative bronchiolitis in murine orthotopic tracheal transplantation. Int Immunopharmacol. 2013;15(4):726-34. doi: http://dx. doi.org/10.1016/j.intimp.2013.03.002.

52. Asthma: Fact sheet $N^{\circ} 307$ : World Health Organization; 2013 [updated November 20137 July 2014]. Available from: http://www.who.int/mediacentre/factsheets/fs307/en/.

53. Aoshiba K, Nagai A. Differences in airway remodeling between asthma and chronic obstructive pulmonary disease. Clin Rev Allergy Immunol. 2004;27 (1):35-43. doi: 10.1385/CRIAl:27:1:035.

54. Conese M, Piro D, Carbone A, Castellani S, Di Gioia S. Hematopoietic and Mesenchymal Stem Cells for the Treatment of Chronic Respiratory Diseases: Role of Plasticity and Heterogeneity. The Scientific World Journal. 2014;2014.

55. Bonfield TL, Koloze M, Lennon DP, Zuchowski B, Yang SE, Caplan Al. Human mesenchymal stem cells suppress chronic airway inflammation in the murine ovalbumin asthma model. Am J Physiol Lung Cell Mol Physiol. 2010;299(6):L760-L70.

56. Nemeth K, Keane-Myers A, Brown JM, Metcalfe DD, Gorham JD, Bundoc VG, et al. Bone marrow stromal cells use TGF- $\beta$ to suppress allergic responses in a mouse model of ragweed-induced asthma. Proceedings of the National Academy of Sciences. 2010;107 (12):5652-7. doi: 10.1073/pnas.091072 0107.

57. Carreras A, Almendros I, Farre R. Potential role of bone marrow mesenchymal stem cells in obstructive sleep apnea. International journal of stem cells. 2011;4(1):43-9. PubMed PMID: 24298333; PubMed Central PMCID: PMC3840967.

58. Carreras A, Almendros I, Acerbi I, Montserrat JM, Navajas D, Farre R. Obstructive apneas induce early release of mesenchymal stem cells into circulating blood. Sleep. 2009;32(1):117-9. PubMed PMID: 19189787; PubMed Central PMCID: PMC 2625316.

59. Carreras A, Rojas M, Tsapikouni T, Montserrat JM, Navajas D, Farre R. Obstructive apneas induce early activation of mesenchymal stem cells and enhancement of endothelial wound healing. Respiratory research. 2010;11:91. doi: 10.1186/1465-9921-11-91. PubMed PMID: 20604943; PubMed Central PMCID: PMC2910004.

60. Carreras A, Almendros I, Montserrat JM, Navajas D, Farré R. Mesenchymal stem cells reduce inflammation in a rat model of obstructive sleep apnea. Respiratory Physiology \& Neurobiology. 2010;172(3): 210-2. doi: http://dx.doi.org/10.1016/j.resp.2010.05. 020.

61. Ramos P, Rubies C, Torres M, Batlle M, Farre R, Brugada J, et al. Atrial fibrosis in a chronic murine model of obstructive sleep apnea: mechanisms and prevention by mesenchymal stem cells. Respiratory research. 2014;15(1):54. PubMed PMID: doi:10.1186/ 1465-9921-15-54. 